\title{
Design and Development of a $\mu$-Synthesis Con- troller for a Flexible Robotic Manipulator, Modelled as a Rotating Euler-Bernoulli Beam
}

\author{
Hamed Moradi, Gholamreza Vossoughi, Firooz Bakhtiari Nejad and Mohammad T. \\ Ahmadian \\ Department of Mechanical Engineering, Sharif \& Amirkabir Universities of Technology, Azadi Street, P.O. Box: \\ 11155-9567, Tehran, Iran
}

\begin{abstract}
(Received 26 September 2012; revised 14 February 2013; accepted 20 March 2013)
In this paper, a robust control strategy for a robotic manipulator, modelled as a cantilever rotating Euler-Bernoulli beam, is developed. Imprecision in the payload mass, unknown properties of the manipulator link, and torque disturbance are included as the sources of uncertainty. The objective is to achieve a desired angular rotation while the vibration of the manipulator tip is suppressed and the control system remains in a stable region. The control input of the system is an external driving torque. For formulation of the continuous system, the mode summation technique is used and equations of motion are described in the Laplace domain. Then, unstructured uncertainties are included in the form of multiplicative input uncertainty. The $\mu$-synthesis control approach is used and an $H_{\infty}$ optimal robust controller is developed based on the DK-iteration algorithm. Results show that the designed controller guarantees the robust stability and performance of the perturbed system against existing uncertainties. Consequently, stability of the closed-loop system, disturbance rejection, and trajectory tracking performance are achieved.
\end{abstract}

\section{INTRODUCTION}

Robotic manipulators are extensively used in areas such as industrial automation, underwater or space vehicles, manufacturing, material handling (e.g., CNC multi-axes milling machines), and medical surgeries. Flexible manipulators have several advantages over their rigid-arm counterparts, such as less material usage (and, consequently, less weight and energy consumption), smaller actuators, and more manoeuvrability. However, due to the inertia and external forces, light structures are more likely to deflect and vibrate. Therefore, the positioning inaccuracy of the end effector causes the performance of the manipulator to be unacceptable. Mechanical stiffening of the components has been used as a traditional solution to avoid such structural vibrations, which has proved to be ineffective for lightweight flexible manipulators. Consequently, the effects of link flexibility must be considered in the dynamic modelling, as stated by De Wit et al. ${ }^{1}$ Thereafter, under external disturbances and parameter variations of the system, effective controllers are required to guarantee the quick and accurate manoeuvrability of manipulators.

Many research studies have been conducted to derive the equations of motion of flexible manipulators and to analyse their dynamics. In the early works, Book, ${ }^{2}$ as well as Low and Vidyasagar, ${ }^{3}$ derived the equations of motion for both rigid and flexible robot manipulators through Lagrangian formulation. To investigate the generic properties of the structural modelling related to the structural control, Spector and Flasher ${ }^{4}$ used distributed sensors on the pinned-free Euler-Bernoulli beam. Choura et al. $^{5}$ found the differential equations describing the planar motion of a rotating, thin, flexible beam. Dynamics of a beam experiencing a combination of rotational and translational motions was analysed by Yuh and Young, ${ }^{6}$ while its finite element simulation was presented by Gaultier and Cleghorn. ${ }^{7}$ Dynamic equations for a planar manipulator with two flexible links, in contact with a constrained surface, have been studied by Matsuno et al. ${ }^{8}$ Using Hamilton's principle, equations of motion for a chain of flexible links have been developed through a systematic procedure by Benati and Morro. ${ }^{9}$ Damaren and Sharf ${ }^{10}$ simulated the constrained motion of flexible-link manipulators, including inertial and geometric nonlinearities.

Fung and Chang ${ }^{11}$ studied the dynamic modelling of a nonlinearly constrained flexible manipulator based on four configurations: Timoshenko, Euler, simple flexure, and rigid body beam theories. A linearised dynamic model for multi-link planar flexible manipulators with an arbitrary number of flexible links has been presented by Chen. ${ }^{12}$ In that linearised model, flexible links were treated as Euler-Bernoulli beams and the Lagrangian approach was used to establish equations of motion. In addition, Meek and Liu ${ }^{13}$ simulated the nonlinear dynamics of flexible manipulators under large overall motions. A study of modelling and the dynamic response of a multistraight-line path tracing a flexible robot manipulator has been done by Kalyoncu ${ }^{14}$ (under the action of an external driving torque and an axial force).

For performance control of the flexible robot manipulators, many control techniques have been developed based on the Euler-Bernoulli beam theory. Point-to-point position control of a flexible beam using the Laplace transform technique has been done by Bhat and Miu. ${ }^{15}$ Diken $^{16}$ investigated the vibration control of the beam using measured shear force as the feedback, and simultaneously achieved the desired angular rotation by using a PD controller. Implementation of a neural network tracking controller for a single flexible link has been compared for PD and PID controllers by Gutierrez et al. ${ }^{17}$ Due 